\title{
Electroacupuncture ameliorates blood-brain barrier dysfunction in 5XFAD Alzheimer's animal model
}

\author{
Mudan Cai, Jun-Hwan Lee, Eun Jin Yang* \\ Department of KM Science Research, Korea Institute of Oriental Medicine, Daejeon 34054, Korea
}

Received: Aug 24, 2021

Revised: Sep 8, 2021

Accepted: Sep 8, 2021

${ }^{*}$ Corresponding author

Eun Jin Yang

Department of KM Science Research,

Korea Institute of Oriental Medicine,

Daejeon 34054, Korea

Tel: +82-42-863-9497

E-mail: yej4823@gmail.com

Copyright $(2021$ Research Institute of Veterinary Medicine, Chungbuk National University. This is an Open Access article distributed under the terms of the Creative Commons Attribution Non-Commercial License (http://creativecommons.org/licenses/ by-nc/4.0/) which permits unrestricted non-commercial use, distribution, and reproduction in any medium, provided the original work is properly cited.

ORCID

Mudan Cai

https://orcid.org/0000-0003-4212-7280

Jun-Hwan Lee

https://orcid.org/0000-0001-5730-6869

Eun Jin Yang

https://orcid.org/0000-0003-3505-7739

Conflict of Interest

The authors declare no potential conflict of interest.

Acknowledgements

This study was supported by the Korea Institute of Oriental Medicine (KIOM) under Grant KSN2021240.

Ethics Approval

The experiments were performed in accordance with the animal care guidelines of the Korea Institute of Oriental Medicine (KIOM) and approved by the Institutional Animal Care and Use Committee (IACUC) of KIOM (approval number 18-033 and 19-011).

\begin{abstract}
Alzheimer's disease $(A D)$ is an irreversible and progressive neurodegenerative disease accompanied by aging, followed by memory impairment and cognitive decline. Although numerous attempts have been made to develop treatments for $A D$, most clinical trials have failed to delay or stop the progression of AD. Electroacupuncture $(E A)$ is a complementary alternative medicine technique widely used to treat pain, inflammation, and neurodegenerative diseases. Additionally, blood-brain barrier (BBB) disruption is a known pathophysiology of neurodegenerative diseases, including AD. Moreover, amyloid beta deposition increases BBB permeability and produces inflammatory cytokines induced by glial activation. However, our previous study revealed that EA treatment at the Taegye acupoints (KI3) improves memory impairment through anti-neuroinflammation and increases glucose metabolism in 5XFAD mice. Therefore, we evaluated whether EA treatment at KI3 regulates BBB dysfunction in the prefrontal cortex of 5XFAD mice. For this study, 6.5-month-old 5XFAD mice were treated with EA stimulation at KI3 three times a week for two weeks. Western blotting, immunohistochemistry, and flow cytometry were used to evaluate the effects of EA treatment on BBB dysfunction. We found that EA stimulation attenuates BBB integrity by protecting BBB tight junction proteins (CD31, aquaporin 4, occludin, and claudin 5) in the prefrontal cortex of 5XFAD mice.

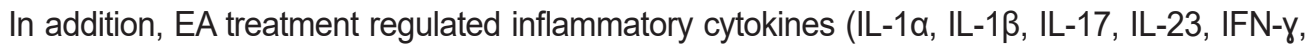
monocyte chemoattractant protein 1 (MCP-1), granulocyte-macrophage colony stimulating factors [GM-CSF], and IL-10) in the peripheral circulation of 5XFAD mice. Therefore, our data suggest that EA treatment could be a therapeutic agent for enhancing BBB dysfunction in AD.
\end{abstract}

Keywords: Alzheimer disease; electroacupuncture; blood-brain barrier; inflammatory cytokines

\section{INTRODUCTION}

Alzheimer's disease (AD) is an irreversible and progressive neurodegenerative disease with the highest prevalence worldwide. The most common early sign is memory loss of recent events [1]. Moreover, the exact cause of $\mathrm{AD}$ remains poorly understood. $\mathrm{AD}$ is a complex disease that has several pathological characterizations, including the formation of amyloid beta (A $\beta$ ) plaques, neurofibrillary tangles, and blood-brain barrier (BBB) dysfunction [2-4]. These are accompanied by 
neuronal and synaptic connectivity impairment, resulting in progressive neuronal cell loss [5, $6]$.

The BBB comprises basement membrane, pericytes, astrocytes, and endothelial cells, tightly connected by tight junctions (TJs) and adherence proteins [7]. The BBB has highly selective permeability by active transport and simultaneously protects the brain from circulating pathogens. Zlokovic et al. suggested that $\mathrm{A} \beta$ deposition in the $\mathrm{AD}$ brains may be due to disruption of homeostasis between its production and clearance from the brain [8]. In addition, $A \beta$ can be eliminated through transport proteins across the BBB, degradation, and perivascular drainage via the vascular membrane [9].

$\mathrm{AD}$ is well known to have significant vascular pathophysiology, including disruption of BBB's integrity and function [7]. For example, A $\beta 42$ leads to increased BBB permeability and decreased the expression level of TJ protein by several mechanisms, such as neuroinflammatory responses, oxidative stress, decreased levels of annexin A1 (an anti-inflammatory messenger) and low-density lipoprotein receptor-related protein 1 (LRP1), and increased levels of receptor for advanced glycation end products (RAGE) and matrix metalloproteinase 9 (MMP9) $[7,10]$. In addition, Wan et al. demonstrated that the oligomer A $\beta 1-42$ is an important damaging factor that results in the upregulation of RAGE expression, finally inducing BBB leakage and disruption of TJ scaffold proteins in $\mathrm{AD}$ [11].

Electroacupuncture (EA) is a complementary alternative medicine technique widely used to treat pain, inflammation, and neurodegenerative diseases in East Asia. EA involves the delivery of an electrical current to the acupoints to stimulate the body. Several studies have demonstrated that EA treatment at acupoints such as Baekoe (GV20), Joksamni (ST36), Daechu (GV14), and Sugu (GV26) decreased infarction volume and improved neurological function by modulating BBB-related protein expression in a focal cerebral ischemia stroke model [12-14]. In addition, GV20 and Yindang (EX-HN3) combined with donepezil could improve learning and memory by regulating BBB permeability in senescence-accelerated mouse prone 8 (SAMP8) mice [15]. Acupuncture treatment at the KI3 acupoint activated cerebral neurons and functional connectivity in elderly patients with mild cognitive impairment through functional magnetic resonance imaging (fMRI) $[16,17]$. In addition, KI3 stimulation regulated brain glucose metabolism in spontaneously hypertensive rats [18]. Furthermore, we demonstrated that EA treatment at KI3 acupoint regulated glucose metabolism in the transient vascular dementia animal model [19] and attenuated memory dysfunction by an increase of anti-neuroinflammation in the 5Xfamilial AD (FAD) model [20].

This study demonstrated the effects of EA treatment at KI3 on BBB integrity, alteration of $\mathrm{TJ}$ scaffold proteins, and inflammatory cytokine levels in peripheral blood circulation in $5 \mathrm{X}$ FAD mice.

\section{MATERIALS AND METHODS}

\section{Animals}

In this study, 5XFAD mice expressing human APP and PSEN1 transgenes were used as AD 
animal models. 5XFAD mice were purchased from the Jackson Laboratory (Bar Harbor, ME, USA) and bred as described previously [20]. DNA was extracted from 1 to $2 \mathrm{~mm}$ tail tissue for genotyping to identify pups using polymerase chain reaction analysis. Four mice per case were housed in specific pathogen-free conditions $\left(21 \pm 3^{\circ} \mathrm{C}, 50 \pm 10 \%, 12\right.$-h light/dark cycle), with ad libitum access to water and food. Female 5XFAD mice used for this experiment were randomly divided into two groups: the 5XFAD transgenic mice $(\mathrm{Tg})$ group and $\mathrm{Tg}$ treated with electroacupuncture (EA) at the KI3 acupoint ( $\mathrm{Tg}+\mathrm{KI}$ ) group. All animal care guidelines of the Korea Institute of Oriental Medicine (KIOM) were adhered to, and all experiments were approved by the Institutional Animal Care and Use Committee of KIOM.

\section{Electroacupuncture (EA) treatment}

EA treatment methods were previously described [20]. The 6.5-month-old 5XFAD mice were stimulated with EA at the Taegye (KI3) acupoint three times a week for two weeks. The $\mathrm{KI} 3$ acupoints were located in the medial aspect of the foot, between the medial malleolus and the Achilles tendon, based on the national standards of the acupoint location (GB12346-90) [20-22]. For EA treatment, 5XFAD mice were anesthetized using isoflurane (Hana Pharm, Hwaseong, Korea), and 8-mm long, 0.18-mm diameter acupuncture needles (Dongbang Medical, Boryeong, Korea) were inserted $2 \mathrm{~mm}$ deep, then an electrical stimulator (Partner-1; Daejeon, Korea) was connected, and the bilateral KI3 acupoints were stimulated. The stimulator run at an intensity of $1 \mathrm{~mA}$ and a frequency of $2 \mathrm{~Hz}$ for $15 \mathrm{~min}$.

\section{Tissue preparation}

Mice were transcardially perfused with phosphate-buffered saline (PBS) for immunofluorescence staining. Whole brains were removed and kept in $4 \%$ paraformaldehyde overnight for fixation, then immersed in a $30 \%$ sucrose for osmotic dehydration at $4^{\circ} \mathrm{C}$. Brain tissues were coronally cut at $30 \mu \mathrm{m}$ thickness for immunostaining using a cryostat (Leica Microtome, Wetzlar, Germany).

The prefrontal cortex was carefully isolated and homogenized in Dulbecco's PBS (DPBS) using a Dounce grinder for western blotting. The homogenate was separated by centrifugation at $2,000 \times \mathrm{g}$ for $5 \mathrm{~min}$ at $4^{\circ} \mathrm{C}$. After centrifugation, the supernatant was removed, and the pellets resuspended in $15 \%(\mathrm{wt} / \mathrm{vol})$ dextran-DPBS, then centrifuged again at $10,000 \times \mathrm{g}$ for 15 min. The supernatant (brain tissue-containing layer) was discarded, and the pellet (microvessel) resuspended in $1 \mathrm{~mL}$ DPBS, transferred to a $20-\mu \mathrm{m}$ cell strainer, and washed with $<10 \mathrm{~mL}$ of DPBS. Finally, the filter was reversed, and microvessels retrieved using 10-20 mL of DPBS and centrifuged at $5,000 \times \mathrm{g}$ for $10 \mathrm{~min}$ to yield the final microvessel pellet. The supernatant was discarded and added $80 \mu \mathrm{L}$ of ice-cold protein lysis buffer (Biosesang, Seongnam, Korea) with inhibitors (Thermo Fisher Scientific, Waltham, MA, USA).

\section{Measurement of serum inflammatory cytokine levels}

To measure inflammatory cytokine levels in mouse serum, whole blood samples were collected from the heart before euthanasia, as previously described [23]. Whole blood was 
allowed to clot at room temperature, separated by centrifugation, and the supernatant was collected. The supernatant was designated as serum and transferred into a new tube for the next experiment. The inflammatory cytokine levels in the serum of mice were measured using LEGEND plex cytokine bead arrays (BioLegend, San Diego, CA, USA). Undiluted serum was used in the experiment, following the manufacturer's instructions. High-performance flow cytometry (BD, East Rutherford, NJ, USA) was used to measure and analyze the data.

\section{Western blots}

The western blotting procedure was used as previously described [20]. The homogenate (20 $\mu \mathrm{g}$ of proteins) was separated on Bolt 4\%-12\% Bis-Tris Plus gels (Thermo Fisher Scientific) and transferred to PVDF membranes. Membranes were incubated in 5\% skim milk for blocking and then incubated in anti-aquaporin 4, occludin, and CD31 antibodies (1:1,000; Abcam, Cambridge, MA, USA) overnight at $4^{\circ} \mathrm{C}$. The next day, the membranes were immersed in indicated secondary antibodies (Santa Cruz Biotechnology, Santa Cruz, CA, USA). Membranes were developed using ChemiDoc Imaging System device (Bio-Rad) for visualization. Images were analyzed using ImageJ (version 1.46j; National Institutes of Health, Bethesda, MD, USA) for quantification.

\section{Immunofluorescence staining}

For immunofluorescence studies, free-floating sections were blocked in bovine serum albumin at room temperature, and then immersed in the indicated primary antibodies: anti-aquaporin 4 (1:1,000; Abcam, Cambridge, MA, USA), anti-CD31 (1:1,000; Abcam), and anti-claudin 5 (1:1,000; Thermo Fisher Scientific) overnight at $4^{\circ} \mathrm{C}$. Then sections were incubated in goat anti-mouse Alexa 488 (1:1,000; Invitrogen, Carlsbad, CA, USA). For visualization, images were acquired using fluorescence microscope (Olympus BX53).

For double immunofluorescence staining, free-floating sections were blocked and incubated in glucose transporter 1 (GLUT1) $\left(1: 1,000\right.$; Abcam) antibody overnight at $4^{\circ} \mathrm{C}$. Sections were immersed in matched secondary goat anti-rabbit Alexa 598 (1:1,000; Invitrogen). After antibody staining, sections were washed in PBS and stained with $1 \%$ thioflavin S for $10 \mathrm{~min}$ to visualize fibrillar $A \beta$. The number of microvessel branch points, microvessels measuring > $60 \mu \mathrm{m}$, and damaged microvessels (near the $\mathrm{A} \beta$ plaques and displayed as disconnected short length forms) were counted per section, per animal, by a researcher blinded to study data.

\section{Statistical analysis}

Statistical comparisons were analyzed using unpaired $t$-test through Prism 9.0 (GraphPad, La Jolla, CA, USA) and bars in figures are presented as the mean \pm SEM.

\section{RESULTS}

Electroacupuncture (EA) treatment attenuates blood-brain barrier (BBB) integrity and decreases damaged microvessels in 5XFAD mice 
Although GLUT1 plays an important role in maintaining BBB integrity, GLUT1 deficiency in endothelial cells induces BBB breakdown in AD mice [24]. In addition, Kook et al. demonstrated that $\mathrm{A} \beta_{1-42}$ disrupts cerebral capillaries and $\mathrm{BBB}$ integrity in 5XFAD mice [25]. Based on these studies, we first determined whether BBB integrity was improved by EA treatment using GLUT1 immunostaining (Fig. 1A). As shown in Fig. 1B and 1C, the number of microvessel branches and microvessels (length $>60 \mu \mathrm{m}$ ) significantly increased by 1.8and 1.6-fold in the EA treatment in the KI3 group compared with that in the non-treated $\mathrm{Tg}$ group in the prefrontal cortex of 5XFAD mice. Kook et al. also observed disconnected short microvessels near $\mathrm{A} \beta$ plaques in $5 \mathrm{XFAD}$ mice [25]. We also confirmed whether EA treatment ameliorates damaged microvessels near $\mathrm{A} \beta$ plaques by co-staining with endothelial markers (GLUT1) and $A \beta_{1-42}$ plaques (Thioflavin S) and found that the number of abnormal microvessels was significantly decreased by 2 -fold in the EA treatment group compared with that in the non-treated Tg group (Fig. 1C). Our results indicate that EA treatment attenuates BBB integrity in the prefrontal cortex of 5XFAD mice.

\section{Electroacupuncture (EA) treatment protects blood-brain barrier (BBB) tight junc- tions in 5XFAD mice}

Previous reports have suggested that $\mathrm{A} \beta$ deposition induces several related proinflammatory and cytotoxic factors in the vasculature of the $\mathrm{AD}$ brain [26, 27]. Additionally, some studies have reported that $A \beta$ deposition regulates TJ-related proteins, including occludins, claudins, and ZO, and finally disrupts BBB's physiological function in $\mathrm{AD}$ patients $[25,28,29]$. There-

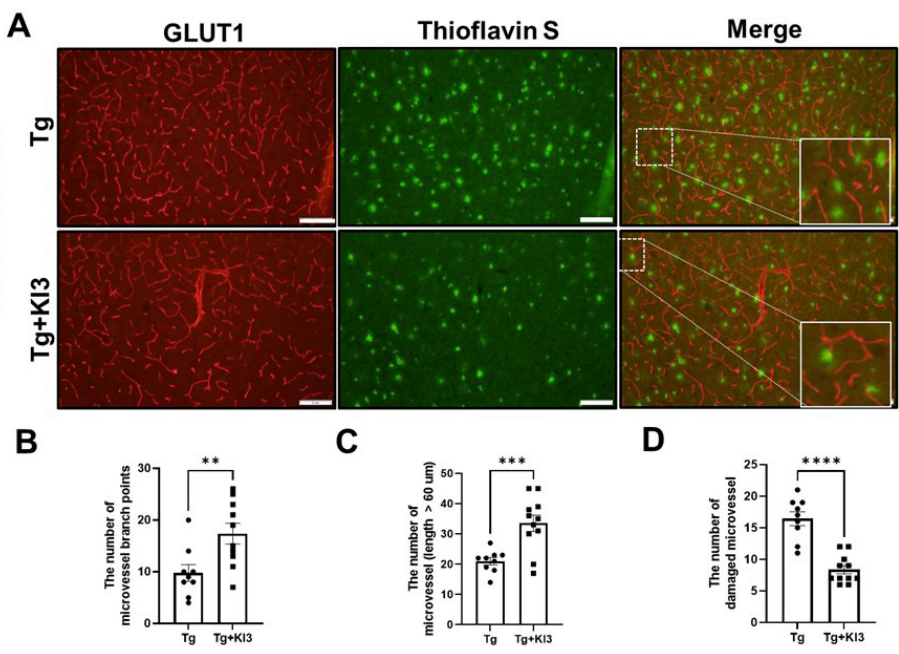

Fig. 1. EA treatment attenuates BBB dysfunction in the prefrontal cortex of 5XFAD mice. Our 6.5-monthold 5XFAD mice received EA treatment at KI3 three times a week for two weeks. (A) The coronal sections of prefrontal cortex region of mice were co-stained with GLUT1 (red, marker of endothelial cell) and Thioflavin $S$ (green, marker of $A \beta_{1-42}$ plaques). Image magnification showing disconnected and damaged microvessels near $A \beta$ plaques in the merged images (Scale bar $=100 \mu \mathrm{m}$ ). (B) Quantitative analysis of the number of microvessel branch points, (C) microvessel (length>60 $\mu \mathrm{m}$ ), and (D) abnormal damaged microvessel in the prefrontal cortex $(n=9-10 /$ group). Data are presented as means \pm SEM $\left({ }^{t+1} p<0.01,{ }^{,+*} p<0.001, \quad p<0.0001\right)$. EA, electroacupuncture; BBB, blood-brain barrier; GLUT1, glucose transporter $1 ; A \beta$, amyloid beta. 
fore, we demonstrated that EA treatment at $\mathrm{KI} 3$ changes BBB TJ proteins in the prefrontal cortex of 5XFAD mice through immunohistochemical staining. First, we determined the intensity of immunostaining for perivascular astrocytic end-feet marker (aquaporin 4), endothelial cell marker (CD31), integral membrane protein, and TJ strand marker (claudin 5). As shown in Fig. 2A and 2B, the levels of BBB TJ-related markers, aquaporin 4, CD31, and claudin 5, were significantly increased by 1.8-, 1.7-, and 2.7-fold in the KI3-EA treatment group compared to those in the non-treated Tg group. We also observed protein expression of BBB TJ-related markers in a microvessel isolated sample of the prefrontal cortex using western blotting. As shown in Fig. 2C and 2D, the group that underwent EA treatment showed significantly increased protein expression of aquaporin 4 and occludin (integral membrane protein) by 1.4- and 9.9-fold, respectively, compared to the non-treated Tg group in the microvessel isolated samples. In addition, the protein expression of CD31 increased by 1.9-fold compared to the non-treated $\mathrm{Tg}$ group , but the difference was not significant. Collectively, these data show that EA treatment at KI3 improves integrity through BBB TJ in the prefrontal cortex of 5XFAD mice.

\section{Electroacupuncture (EA) reduces proinflammatory cytokines in the peripheral cir- culation of 5XFAD mice}

Several reports showed altered levels of several cytokines in the serum of AD mice, including increased proinflammatory cytokines such as IL-1 $\alpha$, IL-1 $\beta$, TNF- $\alpha$, and monocyte chemoattractant protein 1 (MCP-1), and decreased anti-inflammatory cytokine IL-10 [30-32]. In addition, Dubenko et al. showed that IL-17 and IL-23 levels were significantly higher in $\mathrm{AD}$ patients than in controls [33]. Therefore, in this study, we investigated changes in proinflammatory cytokines in the peripheral circulation of 5XFAD mice. Before euthanizing the mice, serum samples were harvested to analyze the level of inflammatory cytokines using flow cytometry (Fig. 3). The results showed that mice who underwent EA treatment at KI3 showed significantly reduced levels of proinflammatory cytokines IL-1a, IL-1 $\beta$, IL-17, IL-23, IFN-r, MCP-1, and GM-CSF by 4.5-, 2.9-, 3.8-, 1.8-, 2.4-, 1.5-, and 3.3-fold, respectively, and dramatically increased IL-10 levels by 2-fold compared to non-treated Tg mice in the serum of 5XFAD mice. Therefore, these data indicate that EA treatment at KI3 regulates pro- and anti-inflammatory cytokines in the peripheral circulation of 5XFAD mice.

\section{DISCUSSION}

The present results demonstrate that EA treatment at the KI3 acupoint attenuates BBB integrity by protecting BBB TJs in the prefrontal cortex of 5XFAD mice. In addition, inflammatory cytokines were regulated by EA treatment at KI3 in the peripheral circulation of 5XFAD mice.

EA treatment has been effectively used to protect cognition via anti-inflammatory and antioxidative stress and to regulate metabolism in AD patients and mice [34-36]. In addition, EA treatment reportedly ameliorates BBB integrity in focal cerebral ischemia and the SAMP8 animal model $[15,37,38]$. A previous study demonstrated that EA treatment at the KI3 acu- 
A
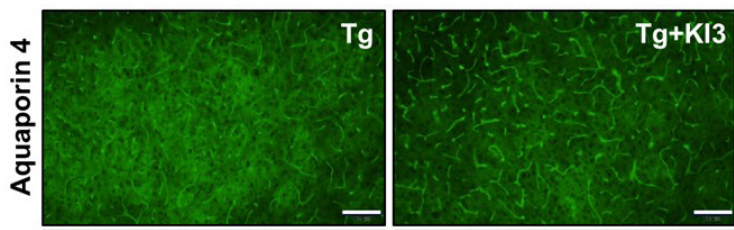

$\operatorname{Tg}$
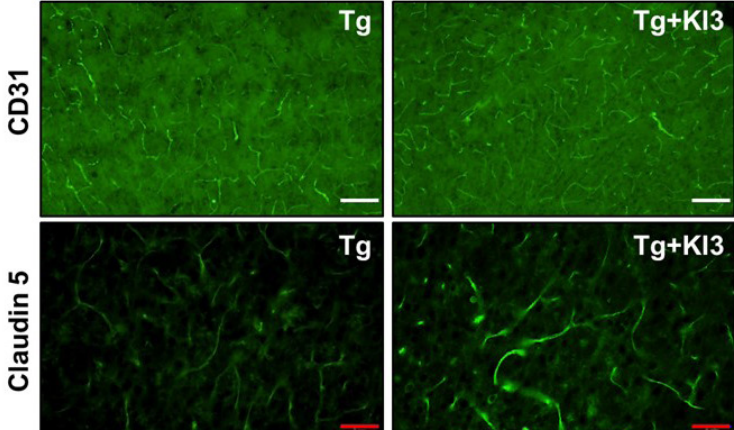

B
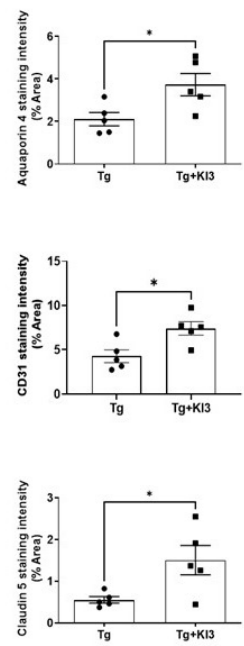

C

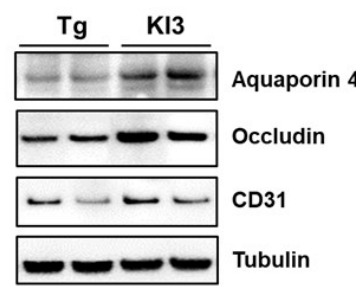

D
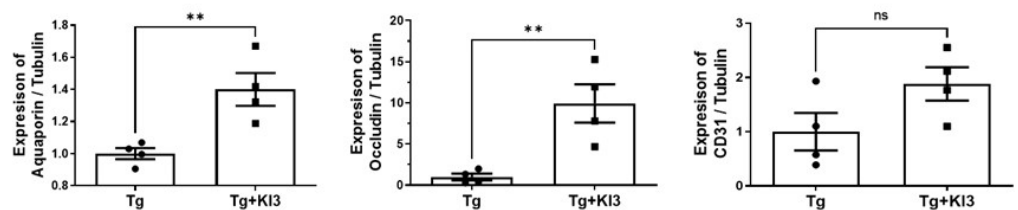

Fig. 2. EA treatment protects BBB tight junction related proteins in the prefrontal cortex of 5XFAD mice. (A) The coronal sections of mice prefrontal cortex region were immunostained with aquaporin 4 (marker of perivascular astrocytic end-feet), CD31 (marker of endothelial cell), and claudin 5 (marker of integral membrane protein) (Scale bar, $100 \mu \mathrm{m}$ for anti-aquoporin 4 and anti-CD31 and $50 \mu \mathrm{m}$ for anti-claudin 5 immunofluorescence staining). (B) Quantitative analysis of the intensity of BBB tight junction related proteins ( $n=5$ / group). (C) Representative western blots for the expression of BBB tight junction related proteins such as aquaporin 4, occludin (marker of integral membrane protein), and CD31 in the microvessel samples isolated from the prefrontal cortex. Tubulin was used as loading control. (D) Quantification of aquaporin 4, occludin, and CD31 levels between $\mathrm{Tg}$ and EA treatment group ( $n=3-4$ I group). Data are normalized to the Tg group and presented as means $\pm \operatorname{SEM}\left({ }^{*} p<0.05,{ }^{* *} p<0.01\right)$. EA, electroacupuncture; BBB, blood-brain barrier.

point improves cognitive impairment through anti-neuroinflammatory effects in 5XFAD mice. Interestingly, we also found that microglia-mediated $A \beta$ degradation decreased by EA treatment at KI3 [20]. Therefore, in this study, we first attempted to investigate the effects of EA treatment at $\mathrm{KI} 3$ on $\mathrm{AD}$-associated $\mathrm{BBB}$ disruption, which appears to be a pathophysiological mechanism in neurodegenerative diseases, including $\mathrm{AD}$ and vascular dementia [39, 40]. We confirmed BBB integrity using GLUT1 immunostaining, where GLUT-1 is limited to expres- 
A

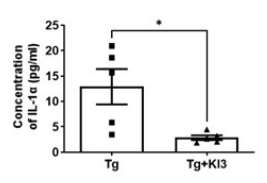

E

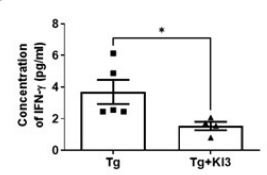

B

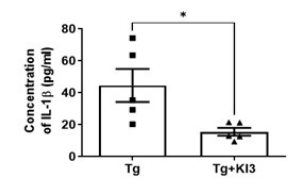

$\mathbf{F}$

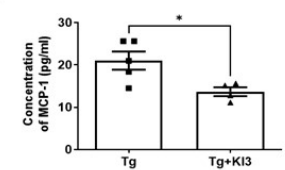

C

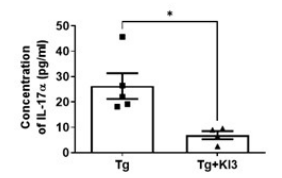

G

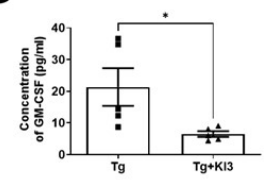

D

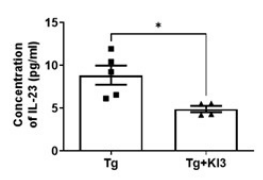

H

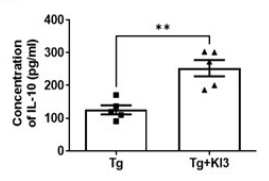

Fig. 3. EA regulates proinflammatory cytokines in 5 XFAD mice's serum. ${ }^{A-H}$ Quantitative analysis of the level of proinflammatory cytokines (IL-1a, IL-1 $\beta$, IL-17, IL-23, IFN-r, MCP-1, and GM-CSF) and antiinflammatory cytokine IL-10 in the serum of 5XFAD mice using flow cytometry. Data are normalized to the Tg group and presented as means \pm SEM (" $p<0.05, "{ }^{\prime} p<0.01$ ). EA, electroacupuncture; MCP-1, monocyte chemoattractant protein 1; GM-CSF, granulocyte-macrophage colony stimulating factors.

sion in endothelial cells and lining the brain microvasculature [25, 41]. We observed that the distribution of $>60-\mu \mathrm{m}$ long individual microvessels and microvessel branch points increased by EA treatment at KI3 (Fig. 1). In addition, previous reports have suggested that abnormal and disrupted cerebral capillaries are present near $\mathrm{A} \beta$ plaques in 5XFAD mice [25]. Based on the above, our data showed that EA treatment significantly decreased the number of damaged microvessels near $\mathrm{A} \beta$. Thus, we concluded that EA treatment ameliorates AD-associated BBB disruption in 5XFAD mice.

Many studies have suggested the relationship between BBB dysfunction and the pathophysiological mechanisms of AD. These results show increased BBB permeability and altered structure of the BBB, including endothelial cells, astrocyte end-feet, pericytes, and basement membrane, in $\mathrm{AD}$ patients and mice [4, 26, 42]. Endothelial cells are the major components of the BBB and connected by TJs, and other neurovascular units contribute to BBB integrity [43]. In addition, Magaki et al. suggested decreased expression of CD31, collagen IV (COL4), and Claudin-5 and increased BBB leakage in AD patients [44]. In addition, Yao et al. demonstrated that the reduction of astrocytic laminin decreases aquaporin-4 and the level of TJ protein and disrupts BBB integrity through the regulation of pericyte differentiation [45]. Moreover, a previous in vitro study suggested that $\mathrm{A} \beta$ deposition decreases $\mathrm{ZO}-1$ and occludin levels and disturbs the organization of claudin- 5 to contribute to BBB function [42]. Based on these previous studies, we found that EA treatment at $\mathrm{KI} 3$ in 5XFAD mice significantly reduced the intensity of aquaporin 4-positive astrocyte end-feet, CD31-positive endothelial cells, claudin 5-positive endothelial TJs, and the protein expression of aquaporin 4, occludin, and CD31 in the prefrontal cortex compared to that in non-treated 5XFAD mice (Fig. 2). Consequently, EA treatment at $\mathrm{KI} 3$ decreased BBB integrity and abnormal microvessels by protecting BBB TJ proteins in $5 \mathrm{XFAD}$ mice.

The BBB is an important physiological barrier between the peripheral circulation and the central nervous system (CNS). Alvarez et al. reported that the levels of IL- $1 \beta$ and TNF- $\alpha$ were increased in the blood of $\mathrm{AD}$ patients [31] and another group demonstrated that the level of 
$\mathrm{BDNF}, \mathrm{TNF} \alpha$, and IL-10 in the serum changed in early or late onset AD [32]. Additionally, $\mathrm{A} \beta$ deposition provides ligands to activate microglial $\mathrm{A} \beta$ phagocytosis, while also producing microglia-mediated proinflammatory cytokines [46]. IFN cytokines play an important role in activating microglial responses in $\mathrm{AD}$ and aging [47]. Yamamoto et al. indicated that IFN- $\gamma$ induces microglial TNF- $\alpha$, and enhance A $\beta$ deposition through BACE1 expression and suppression of $A \beta$ clearance in in vitro studies [48]. In addition, Steeland et al. demonstrated that TNF receptor 1-deficient APP/PS1 mice ameliorated A $\beta$ levels and inflammatory factor expression, improved CSF-blood barrier integrity, and improved memory [49]. In addition, blocking the IL-1 cascade ameliorates brain inflammatory responses, cognition, and tau pathology, and partially reduces certain fibrillar and oligomeric forms of $A \beta$. In addition, Choi et al. demonstrated that normal human astrocytes express several cytokines and chemokines, including IL-6, granulocyte and granulocyte-macrophage colony stimulating factors (GM-CSF), and MCP-1/ CCL2. Additionally, activated astrocytes by IL-1 $\beta$ and TNF- $\alpha$ were the newly secreted IL-1 $\beta$, IL-1ra, TNF- $\alpha$, and MIP-1 $\alpha$ (CCL3) [50]. In addition, astrocyte stimulation with IL-17 increases proinflammatory cytokines and neutrophil-mobilizing cytokines and chemokines, including GM-CSF, CXCL2 (MIP-2), MMP9 [51]. Furthermore, activated microglia and astrocytes release inflammatory cytokines (CXCL8/IL-8, CCL2/MCP-1, TNF-alpha, IL-1beta/IL1F2), leading to peripheral immune cell entry into the CNS. Leukocytes, a circulating immune cell, migrate through the $\mathrm{BBB}$ into the $\mathrm{AD}$ brain to secrete inflammatory cytokines and trigger increased BBB permeability [26]. Therefore, inflammatory cytokines are strongly related to neuroinflammation induced by glial cell activation in the CNS and peripheral nervous system (PNS). Interestingly, our previous data showed that EA treatment of KI3 significantly reduced microglia and astrocytes in the prefrontal cortex of 5XFAD mice. In addition, the number of colocalized $\mathrm{A} \beta_{1-42}$ and microglia was dramatically reduced by EA treatment in the prefrontal cortex of 5XFAD mice [20]. The present results showed significantly reduced serum levels of inflammatory cytokines (IL-1 $\alpha$, IL-1 $\beta$, IL-17, IL-23, IFN-y, MCP-1, GM-CSF, and IL-10) after EA treatment in 5XFAD mice using flow cytometry. This means that EA treatment significantly regulates inflammatory cytokines in the CNS and PNS to protect BBB integrity and BBB TJs.

In summary, our data suggest that EA treatment at KI3 improves BBB integrity and decreases abnormal microvessels by increasing BBB TJ proteins. Additionally, inflammatory cytokines were regulated by EA treatment in the peripheral circulation of 5XFAD mice. Therefore, we suggest that EA treatment at KI3 could be a potential therapeutic agent for enhancing $\mathrm{BBB}$ integrity in neurodegenerative diseases. However, this study has some limitations. EA treatment parameters such as frequency and intensity should be confirmed to achieve better treatment outcomes. In addition, the molecular mechanism of BBB integrity and influx of peripheral immune cells into the CNS should be investigated by EA treatment.

\section{REFERENCES}

1. Burns A, Iliffe S. Alzheimer's disease. Br Med J 2009;338:b158. 
2. Huang HC, Jiang ZF. Accumulated amyloid- $\beta$ peptide and hyperphosphorylated tau protein: relationship and links in Alzheimer's disease. J Alzheimers Dis 2009;16:15-27.

3. Breijyeh Z, Karaman R. Comprehensive review on Alzheimer's disease: causes and treatment. Molecules 2020;25:5789.

4. Sweeney MD, Sagare AP, Zlokovic BV. Blood-brain barrier breakdown in Alzheimer disease and other neurodegenerative disorders. Nat Rev Neurol 2018;14:133-150.

5. Tackenberg C, Kulic L, Nitsch RM. Familial Alzheimer's disease mutations at position 22 of the amyloid $\beta$-peptide sequence differentially affect synaptic loss, tau phosphorylation and neuronal cell death in an ex vivo system. PLOS ONE 2020;15:e0239584.

6. Querfurth HW, LaFerla FM. Alzheimer's disease. N Engl J Med 2010;362:329-344.

7. Park JC, Baik SH, Han SH, Cho HJ, Choi H, Kim HJ, Choi H, Lee W, Kim DK, Mook-Jung I. Annexin A1 restores $A \beta_{1-42}$-induced blood-brain barrier disruption through the inhibition of RhoA-ROCK signaling pathway. Aging Cell 2017;16:149-161.

8. Zlokovic BV, Yamada S, Holtzman D, Ghiso J, Frangione B. Clearance of amyloid $\beta$-peptide from brain: transport or metabolism? Nat Med 2000;6:718.

9. Bell RD, Zlokovic BV. Neurovascular mechanisms and blood-brain barrier disorder in Alzheimer's disease. Acta Neuropathol 2009;118:103-113.

10. Cai Z, Qiao PF, Wan CQ, Cai M, Zhou NK, Li Q. Role of blood-brain barrier in Alzheimer's disease. J Alzheimers Dis 2018;63:1223-1234.

11. Wan W, Cao L, Liu L, Zhang C, Kalionis B, Tai X, Li Y, Xia S. A $\beta_{1-42}$ oligomer-induced leakage in an in vitro blood-brain barrier model is associated with up-regulation of RAGE and metalloproteinases, and down-regulation of tight junction scaffold proteins. J Neurochem 2015; 134:382-393.

12. Xu H, Zhang Y, Sun H, Chen S, Wang F. Effects of acupuncture at GV20 and ST36 on the expression of matrix metalloproteinase 2, aquaporin 4, and aquaporin 9 in rats subjected to cerebral ischemia/reperfusion injury. PLOS ONE 2014;9:e97488.

13. Jung YS, Lee SW, Park JH, Seo HB, Choi BT, Shin HK. Electroacupuncture preconditioning reduces ROS generation with NOX4 down-regulation and ameliorates blood-brain barrier disruption after ischemic stroke. J Biomed Sci 2016;23:32.

14. Lin XM, Chen LP, Yao X. The impact of different duration of EA-pretreatment on expression of MMP-9 and VEGF in blood-brain barrier in rats with cerebral ischemia-reperfusion injury. Zhen Ci Yan Jiu 2015;40:40-44.

15. Yang ZX, Tang CL, Li XH, Zhu ZW, Qiu L, An HY, Wu MJ, Yang YH. Effect of electroacupuncture combined with Donepezil on learning-memory ability and expression of hippocampal $\beta$-amyloid clearance-related genes in SAMP8 mice. Zhen Ci Yan Jiu 2020;45:281-286.

16. Feng Y, Bai L, Ren Y, Chen S, Wang H, Zhang W, Tian J. FMRI connectivity analysis of acupuncture effects on the whole brain network in mild cognitive impairment patients. Magn Reson Imaging 2012;30:672-682.

17. Chen S, Xu M, Li H, Liang J, Yin L, Liu X, Jia X, Zhu F, Wang D, Shi X, Zhao L. Acupuncture at the Taixi (KI3) acupoint activates cerebral neurons in elderly patients with mild cognitive impairment. Neural Regen Res 2014;9:1163-1168. 
18. Li J, Peng C, Lai D, He K, Wang Y, Zhang G, Wu Y, Nie B, Shan B, Tang C, Lai X. Changes in cerebral glucose metabolism after acupuncture at KI3 in spontaneously hypertensive rats: a positron emission tomography study. Acupunct Med 2019;37:107-115.

19. Yang EJ, Cai M, Lee JH. Neuroprotective effects of electroacupuncture on an animal model of bilateral common carotid artery occlusion. Mol Neurobiol 2016;53:7228-7236.

20. Cai M, Lee JH, Yang EJ. Electroacupuncture attenuates cognition impairment via anti-neuroinflammation in an Alzheimer's disease animal model. J Neuroinflammation 2019;16:264.

21. Lu KW, Yang J, Hsieh CL, Hsu YC, Lin YW. Electroacupuncture restores spatial learning and downregulates phosphorylated N-methyl-D-aspartate receptors in a mouse model of Parkinson's disease. Acupunct Med 2017;35:133-141.

22. Zhu B, Wang Y, Zhang G, Ouyang H, Zhang J, Zheng Y, Zhang S, Wu C, Qu S, Chen J, Huang Y, Tang C. Acupuncture at KI3 in healthy volunteers induces specific cortical functional activity: an fMRI study. BMC Complement Altern Med 2015;15:361.

23. Cai M, Lee KW, Choi SM, Yang EJ. TDP-43 modification in the hSOD $1^{\mathrm{G} 93 \mathrm{~A}}$ amyotrophic lateral sclerosis mouse model. Neurol Res 2015;37:253-262.

24. Winkler EA, Nishida Y, Sagare AP, Rege SV, Bell RD, Perlmutter D, Sengillo JD, Hillman S, Kong P, Nelson AR, Sullivan JS, Zhao Z, Meiselman HJ, Wenby RB, Soto J, Abel ED, Makshanoff J, Zuniga E, De Vivo DC, Zlokovic BV. GLUT1 reductions exacerbate Alzheimer's disease vasculo-neuronal dysfunction and degeneration. Nat Neurosci 2015;18:521-530.

25. Kook SY, Hong HS, Moon M, Ha CM, Chang S, Mook-Jung I. A $\beta 1-42-R A G E$ interaction disrupts tight junctions of the blood-brain barrier via $\mathrm{Ca}^{2+}$-calcineurin signaling. J Neurosci 2012;32:8845-8854.

26. Zenaro E, Piacentino G, Constantin G. The blood-brain barrier in Alzheimer's disease. Neurobiol Dis 2017;107:41-56.

27. Carrano A, Hoozemans JJM, van der Vies SM, Rozemuller AJM, van Horssen J, de Vries HE. Amyloid beta induces oxidative stress-mediated blood-brain barrier changes in capillary amyloid angiopathy. Antioxid Redox Signal 2011;15:1167-1178.

28. Marco S, Skaper SD. Amyloid $\beta$-peptide ${ }_{1-42}$ alters tight junction protein distribution and expression in brain microvessel endothelial cells. Neurosci Lett 2006;401:219-224.

29. Yu X, Ji C, Shao A. Neurovascular unit dysfunction and neurodegenerative disorders. Front Neurosci 2020;14:334.

30. Italiani P, Puxeddu I, Napoletano S, Scala E, Melillo D, Manocchio S, Angiolillo A, Migliorini P, Boraschi D, Vitale E, Di Costanzo A. Circulating levels of IL-1 family cytokines and receptors in Alzheimer's disease: new markers of disease progression? J Neuroinflammation 2018; $15: 342$.

31. Alvarez XA, Franco A, Fernández-Novoa L, Cacabelos R. Blood levels of histamine, IL$1 \beta$, and TNF- $\alpha$ in patients with mild to moderate Alzheimer disease. Mol Chem Neuropathol 1996;29:237-252.

32. Gezen-Ak D, Dursun E, Hanağası H, Bilgiç B, Lohman E, Araz ÖS, Atasoy İL, Alaylığlu M, Önal B, Gürvit H, Yılmazer S. BDNF, TNF $\alpha$, HSP90, CFH, and IL-10 serum levels in patients with early or late onset Alzheimer's disease or mild cognitive impairment. J Alzheimers Dis 
2013;37:185-195.

33. Dubenko OE, Chyniak OS, Potapov OO. Levels of proinflammatory cytokines Il-17 and Il-23 in patients with Alzheimer's disease, mild cognitive impairment and vascular dementia. Wiad Lek 2021;74:68-71.

34. Li K, Shi G, Zhao Y, Chen Y, Gao J, Yao L, Zhao J, Li H, Xu Y, Chen Y. Electroacupuncture ameliorates neuroinflammation-mediated cognitive deficits through inhibition of NLRP3 in presenilin1/2 conditional double knockout mice. Neural Plast 2021;2021:8814616.

35. Song YY, Xu WT, Zhang XC, Ni GX. Mechanisms of electroacupuncture on Alzheimer's disease: a review of animal studies. Chin J Integr Med 2020;26:473-480.

36. Xu A, Zeng Q, Tang Y, Wang X, Yuan X, Zhou Y, Li Z. Electroacupuncture protects cognition by regulating tau phosphorylation and glucose metabolism via the AKT/GSK3 $\beta$ signaling pathway in Alzheimer's disease model mice. Front Neurosci 2020;14:585476.

37. Dong H, Fan YH, Zhang W, Wang Q, Yang QZ, Xiong LZ. Repeated electroacupuncture preconditioning attenuates matrix metalloproteinase- 9 expression and activity after focal cerebral ischemia in rats. Neurol Res 2009;31:853-858.

38. Zhang J, Lin X, Zhou H, Chen Y, Xiao S, Jiao J, Zhao Y, Di Z. Electroacupuncture: a new approach to open the blood-brain barrier in rats recovering from middle cerebral artery occlusion. Acupunct Med 2018;36:377-385.

39. Michalicova A, Majerova P, Kovac A. Tau protein and its role in blood-brain barrier dysfunction. Front Mol Neurosci 2020;13:570045.

40. Kamat PK, Swarnkar S, Rai S, Kumar V, Tyagi N. Astrocyte mediated MMP-9 activation in the synapse dysfunction: an implication in Alzheimer disease. Ther Targets Neurol Dis 2014;1:e243.

41. Kalaria RN. The blood-brain barrier and cerebrovascular pathology in Alzheimer's disease. Ann N Y Acad Sci 1999;893:113-125.

42. Desai BS, Monahan AJ, Carvey PM, Hendey B. Blood-brain barrier pathology in Alzheimer's and Parkinson's disease: implications for drug therapy. Cell Transplant 2007;16:285-299.

43. Liu WY, Wang ZB, Zhang LC, Wei X, Li L. Tight junction in blood-brain barrier: an overview of structure, regulation, and regulator substances. CNS Neurosci Ther 2012;18:609-615.

44. Magaki S, Tang Z, Tung S, Williams CK, Lo D, Yong WH, Khanlou N, Vinters HV. The effects of cerebral amyloid angiopathy on integrity of the blood-brain barrier. Neurobiol Aging 2018;70:70-77.

45. Yao Y, Chen ZL, Norris EH, Strickland S. Astrocytic laminin regulates pericyte differentiation and maintains blood brain barrier integrity. Nat Commun 2014;5:3413.

46. Tejera D, Heneka MT. Microglia in neurodegenerative disorders. Methods Mol Biol 2019; 2034:57-67.

47. Gorlé N, Vandenbroucke RE. Interferons: a molecular switch between damage and repair in ageing and Alzheimer's disease. Mech Ageing Dev 2019;183:111148.

48. Yamamoto M, Kiyota T, Horiba M, Buescher JL, Walsh SM, Gendelman HE, Ikezu T. Interferon- $\gamma$ and tumor necrosis factor- $\alpha$ regulate amyloid- $\beta$ plaque deposition and $\beta$-secretase expression in Swedish mutant APP transgenic mice. Am J Pathol 2007;170:680-692. 
49. Steeland S, Gorlé N, Vandendriessche C, Balusu S, Brkic M, Van Cauwenberghe C, Van Imschoot G, Van Wonterghem E, De Rycke R, Kremer A, Lippens S, Stopa E, Johanson CE, Libert C, Vandenbroucke RE. Counteracting the effects of TNF receptor-1 has therapeutic potential in Alzheimer's disease. EMBO Mol Med 2018;10:e8300.

50. Choi SS, Lee HJ, Lim I, Satoh J, Kim SU. Human astrocytes: secretome profiles of cytokines and chemokines. PLOS ONE 2014;9:e92325.

51. Kang Z, Altuntas CZ, Gulen MF, Liu C, Giltiay N, Qin H, Liu L, Qian W, Ransohoff RM, Bergmann C, Stohlman S, Tuohy VK, Li X. Astrocyte-restricted ablation of interleukin-17-induced Act1-mediated signaling ameliorates autoimmune encephalomyelitis. Immunity 2010; $32: 414-425$. 